\title{
Distorted Wave Emission Function (DWEF) Calculations of RHIC HBT and Spectra
}

\author{
John G. Cramer ${ }^{1}$ and Gerald A. Miller ${ }^{1}$ \\ ${ }^{1}$ Department of Physics, Box 351560, University of Washington, Seattle, Washington 98195-1560 USA
}

Received on 23 February, 2007

\begin{abstract}
The emission of pions produced within a dense, strongly-interacting system of matter in the presence of strong radial flow and absorption is described using a relativistic optical model formalism, replacing the attenuated or unattenuated plane waves of earlier emission function approaches with "distorted wave" solutions to a relativistic wave equation including a complex optical potential. The resulting distorted-wave emission function model (DWEF) is used in numerical calculations to fit HBT correlations and the resonance-corrected pion spectrum from central-collision STAR Au+Au pion data at $\sqrt{s}=200 \mathrm{GeV}$. Excellent agreement with the STAR data are obtained. This allows us to predict HBT radii over a range of centralities for both $\mathrm{Au}+\mathrm{Au}$ and $\mathrm{Cu}+\mathrm{Cu}$ collisions.
\end{abstract}

Keywords: Distorted wave emission function; Relativistic heavy ion collisions; Optical model; Chiral symmetry restoration

\section{INTRODUCTION}

Many of the "signals" from analysis of $\mathrm{Au}+\mathrm{Au}$ collisions at RHIC suggest that a quark gluon plasma (QGP) has been created in the initial stages of the collision. A major problem with such interpretations has been that a QGP scenario would require a large source that has expanded for a long time before freeze out and has a long duration for emission of pions. On the other hand, analysis of RHIC data using HBT interferometry has been interpreted as indicating a relatively small unexpanded source with a very short pion emission duration.

In an effort to understand the origins of this problem, we have taken a new approach to RHIC physics. We have attempted to describe the transport of pions starting at chemical freezeout within the hot dense medium of the collision, including the effects of opacity, refraction, and wavelike behavior, by using quantum wave mechanics and the nuclear optical model, as reformulated for the "Bjorken tube" collision geometry. We have produced a relativistic quantum mechanical description of the collision medium that includes collective flow as well as pion absorption and refraction in a complex potential. We use conventional "hydro-inspired" emission function, but we interpret it as describing the production of pions at chemical (rather than kinetic) freezeout. We combine this with pion distorted waves produced by solving the Klein-Gordon wave equation for pions propagating in the post-collision medium. The wave functions are generated using the Numerov algorithm and are used to calculate multidimensional overlap integrals providing predictions of pion momentum spectra and HBT radii.

\section{FORMALISM OF THE DWEF MODEL}

Extensive details of the formalism used in our model have been presented in a long paper to be published in Journal of Physics G [1], and the formal approach will only be summarized here. Briefly, we treat the dynamics of the observed pions from their point of initial emission at chemical freezeout. We separate the emitted particles into "channels" and explicitly treat only the channel including those pions participating in Bose-Einstein symmetrization and leading to the observed

\section{HBT correlation "bump".}

We apply a nuclear-type optical model to the pions in that channel as they traverse the hot dense medium of the collision fireball and emerge into the vacuum. We deal with other channels of the problem (e.g., halo pions, pions from long-lived resonances, reaction-channel absorption of pions, ...) through the use of an imaginary potential that removes pions from the channel of interest. We solve the Klein-Gordon wave equation with the Numerov algorithm in a partial-wave expansion and numerically calculate the wave functions of the pions in the channel of interest. We do not explicitly employ a freezeout hypersurface, but rather allow the optical potential to describe the interactions of the emitted pions with the medium.

We employ a "hydro-inspired" multi-dimensional Gaussian emission function $S_{0}(\vec{x}, \vec{p})$ to describe the probability of pion emission as a function of position and momentum in the medium. We then combine this with optical model wave functions to obtain $S(\vec{x}, \vec{p})$, a distorted-wave emission function (DWEF) that is used to calculate the pion correlation function and spectrum. The optical potential used is not explicitly time dependent, but it acts only over a relatively short time interval that is sharply limited by the emission function.

The model uses twelve parameters, as follows. The two thermal parameters are the emission temperature $(T)$ and the pion chemical potential $\left(\mu_{\pi}\right)$. The two space parameters are the medium radius $\left(R_{W S}\right)$ and diffuseness $\left(a_{W S}\right)$ characterizing a Woods-Saxon-squared matter distribution. The two time parameters are the expansion duration $\left(\tau_{0}\right)$ and emission duration $(\Delta \tau)$. The two flow parameters are the maximum radial flow rapidity $\left(\eta_{f}\right)$ and longitudinal flow $(\Delta \eta)$. The three optical model potential parameters are the real $\left(\operatorname{Re}\left(w_{2}\right)\right)$ and imaginary $\left(\operatorname{Im}\left(w_{2}\right)\right)$ parts of the momentumsquared-dependent potential and the real part $\left(\operatorname{Re}\left(w_{0}\right)\right)$ of the momentum-independent potential. The model also includes the strength $(\varepsilon)$ of the Kisslinger-type gradient term in the wave equation, but we have usually taken $\varepsilon=0$. In most searches, the pion chemical potential for best fitting has been found to be near $140 \mathrm{MeV}$, so we have reduced the number of fitting parameters by setting the pion chemical potential to the charged pion mass $\left(\mu_{\pi}=m_{\pi}\right)$. 
TABLE I: Best fit parameters used in the calculations shown, with variances. Bold-face indicates parameters not varied in the fitting procedure (see text)

\begin{tabular}{|ccccccccccc|}
\hline $\begin{array}{c}T \\
(\mathrm{MeV})\end{array}$ & $\eta_{f}$ & $\begin{array}{c}\Delta \tau \\
(\mathrm{fm} / \mathrm{c})\end{array}$ & $\begin{array}{c}R_{W S} \\
(\mathrm{fm})\end{array}$ & $\begin{array}{c}a_{W S} \\
(\mathrm{fm})\end{array}$ & $\begin{array}{c}w_{0} \\
\left(\mathrm{fm}^{-2}\right)\end{array}$ & $w_{2}$ & $\begin{array}{c}\tau_{0} \\
(\mathrm{fm} / \mathrm{c})\end{array}$ & $\Delta \eta$ & $\varepsilon$ & $\begin{array}{c}\mu_{\pi} \\
(\mathrm{MeV})\end{array}$ \\
\hline 156.58 & 1.310 & 2.0731 & 11.867 & 1.277 & 0.0693 & $0.856+i 0.116$ & 9.04 & 1.047 & $\mathbf{0 . 0 0 0}$ & $\mathbf{1 3 9 . 5 7}$ \\
& \pm 0.025 & \pm 0.07 & \pm 0.06 & \pm 0.015 & \pm 0.046 & $\pm 0.014 \pm 0.002$ & \pm 0.10 & \pm 0.032 & & \\
\hline
\end{tabular}

\section{APPLICATIONS OF THE DWEF MODEL}

Numerical calculations applying this formalism were placed under the control of a Marquardt-Levenberg chisquared minimization program that varied up to 12 model parameters to obtain the best fit to STAR $\sqrt{s_{N N}}=200 \mathrm{GeV}$ $\mathrm{Au}+\mathrm{Au}$ pion spectrum [3] and HBT radii [4]. We note that we calculate the HBT radii by explicitly evaluating the correlation function near its half-maximum point and calculating the Gaussian radius that would give this value. We find that this method gives stable results even in the region of low average momentum $K$, while we have found that the widely-used second-moment method is unreliable in that region.

We note that previously published descriptions of this work [2] solved the Klein-Gordon equation with Runge-Kutta integration using routines inherited from older well-tested pion codes. The step-size stability of the calculations had been carefully checked for variation of the HBT radii values, and a "stable" step size of $0.017 \mathrm{fm}$ was used in all searches. However, it was recently discovered that the slope of the predicted pion momentum spectrum depended on subtle cancellations among wave functions that were strongly dependent to the integration step size used in wave equation solution. Consequently, the spectrum slope progressively decreased with decreasing step size, with an extremely small step size (0.001 $\mathrm{fm}$ or less) required for spectrum slope stability. Calculations with such a small step size require many computation hours to make a single prediction, invite problems with roundoff errors, and are not useful in parameter searches. The consequence of this problem was that the fit parameter values obtained in previous searches were incorrect because of an unphysical bias that favored large values of the emission temperature and flow. This problem was eliminated by using the more numerically stable and convergent Numerov algorithm to solve the wave equation. This procedure provided fast and reliable predictions at integration step sizes around $0.02 \mathrm{fm}$. The STAR data was refitted, leading to the new results presented here. Eliminating this problem reduced fitted source temperatures from around $200 \mathrm{MeV}$ to around $160 \mathrm{MeV}$.

To illustrate the importance of the various effects of the DWEF model for computing the radii $R_{O}, R_{S}$ and the spectrum, we have done calculations using the best fit parameters with various effects switched on separately. This is shown in Fig. 1. The curves labelled DWEF show the full calculation. Those labelled PWEF are computed using plane waves, i.e., the optical potential $\left(w_{0,2}\right)$ and flow $\left(\eta_{F}\right)$ are set to zero. The curves labelled $\operatorname{Re}\left(w_{0}\right)$ only and $\operatorname{Re}\left(w_{2}\right)$ only use only the real constant or momentum-dependent parts of the opti- cal potential, respectively, set to the Table 1 values, with no flow. The curves labelled $\operatorname{Im}\left(w_{2}\right)$ only use only the imaginary momentum-dependent part of the optical potential set to the Table 1 value, with no flow. Finally, the curves labelled flow uses the Table 1 value of $\eta_{F}$, with no optical potential. All other parameters in these calculations are set to the best fit values of Table I. This study indicates that both flow and the optical potential modify the HBT radii, but only the momentum dependent parts of the optical potential $\left(w_{2}\right)$ affect the spectrum.

Table 1 gives the parameters of our best fit to the STAR data for $\mathrm{Au}+\mathrm{Au}$ central collisions at $200 \mathrm{GeV}$. Table I also gives the estimated variances of those fit parameters that were varied, as calculated by determining the parameter variation required to increase the $\chi^{2}$ value by one unit. Correlations between different parameters are not considered. The parameters shown in bold face were set to the values indicated. For this fit to the data, as shown in Figs. 2 and 3, the $\chi^{2}$ is 89.63, and the $\chi^{2}$ per degree of freedom is 4.07 . We note that the emission temperature preferred by the fit, $T=156.58 \mathrm{MeV}$, is significantly smaller than that of previous reports because the slope problem mentioned above has been corrected, and that it roughly corresponds to estimates of the chemical freezeout temperature.

Figs. 1 and 2 show DWEF calculations using the best fit of Table I as compared with the STAR data $[3,4]$ used in the fit. We see that both the HBT radii and the spectrum (including the spectrum normalization) are reproduce very well indeed. However, some clarification is needed here on the issue of resonance pions. Our model describes only those "direct" pions that are emitted directly within the fireball and that participate fully in the HBT correlation. It does not predict the "halo" fraction of pions originating from resonance decays later in the process that will not participate in the HBT correlation (or that would make an unmeasurable "spike" in the correlation function near $q=0$ ). These latter pions are present in the measured spectrum and must be removed before fitting the spectrum with a DWEF calculation. We do this by using square root of the HBT $\lambda$ parameter as an estimate of the direct pion fraction, correct the spectrum for fitting with this, and "uncorrect" the fit for comparison with the measured spectrum.

\section{NON-CENTRAL GOLD-GOLD AND COPPER-COPPER COLLISIONS}

Our analysis so far has focused on the central (0-5\%) STAR $\sqrt{S_{N N}}=200 \mathrm{GeV} \mathrm{Au}+\mathrm{Au}$ data. However, the STAR collaboration performed measurements of pion correlations and spectra 

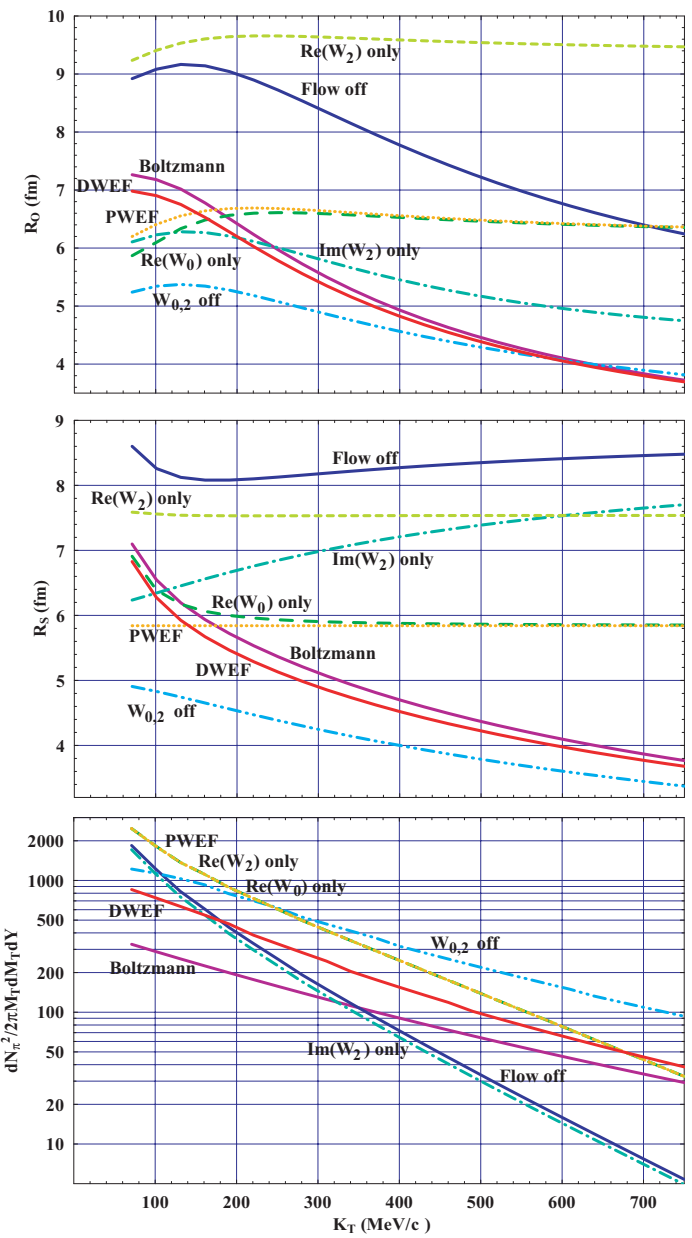

FIG. 1: (Color online) Calculations of $R_{O}, R_{S}$, and spectrum, isolating potential and flow effects. DWEF (red solid) full calculation; PWEF (orange dotted) plane wave calculation with no optical potential or flow; $\operatorname{Re}\left(w_{2}\right)$ only (green dashed); $\operatorname{Im}\left(w_{2}\right)$ only (solid violet) calculation with no flow and only real part of momentum dependent optical potential $\left(w_{2}\right) ; \operatorname{Re}\left(w_{0}\right)$ only (green dashed) calculation with no flow and only real constant optical potential $\left(w_{0}\right)$; flow turned off (blue solid) calculation with flow but no optical potential; $w_{0,2}$ off (turquoise dashed double dot) optical potential turned off; substituting Boltzmann for Bose-Einstein thermal distribution (violet solid). Non-zero parameters in all calculations have the values of Table I.

at $\sqrt{S_{N N}}=200 \mathrm{GeV} \mathrm{Au}+\mathrm{Au}$ and $\mathrm{Cu}+\mathrm{Cu}$ as a function of centrality.

For non-central events, our optical potential would depend on the direction of impact parameter $b$ as well as its magnitude. The simple dependence on the $b$ was exploited heavily in previous sections to simplify the calculations, so, in principle, non-central collisions are not a part of our model. However, we can make simplifying assumptions that can allow us to predict the observables for non-central collisions. In particular, we assume that a non-central collision resembles a central collision with the same number of participants. This assumption allows us to extrapolate our results to systems that do not have perfect centrality by using participant scaling. In particular, we take the space-time parameters $R_{W S}, a_{W S}$, and
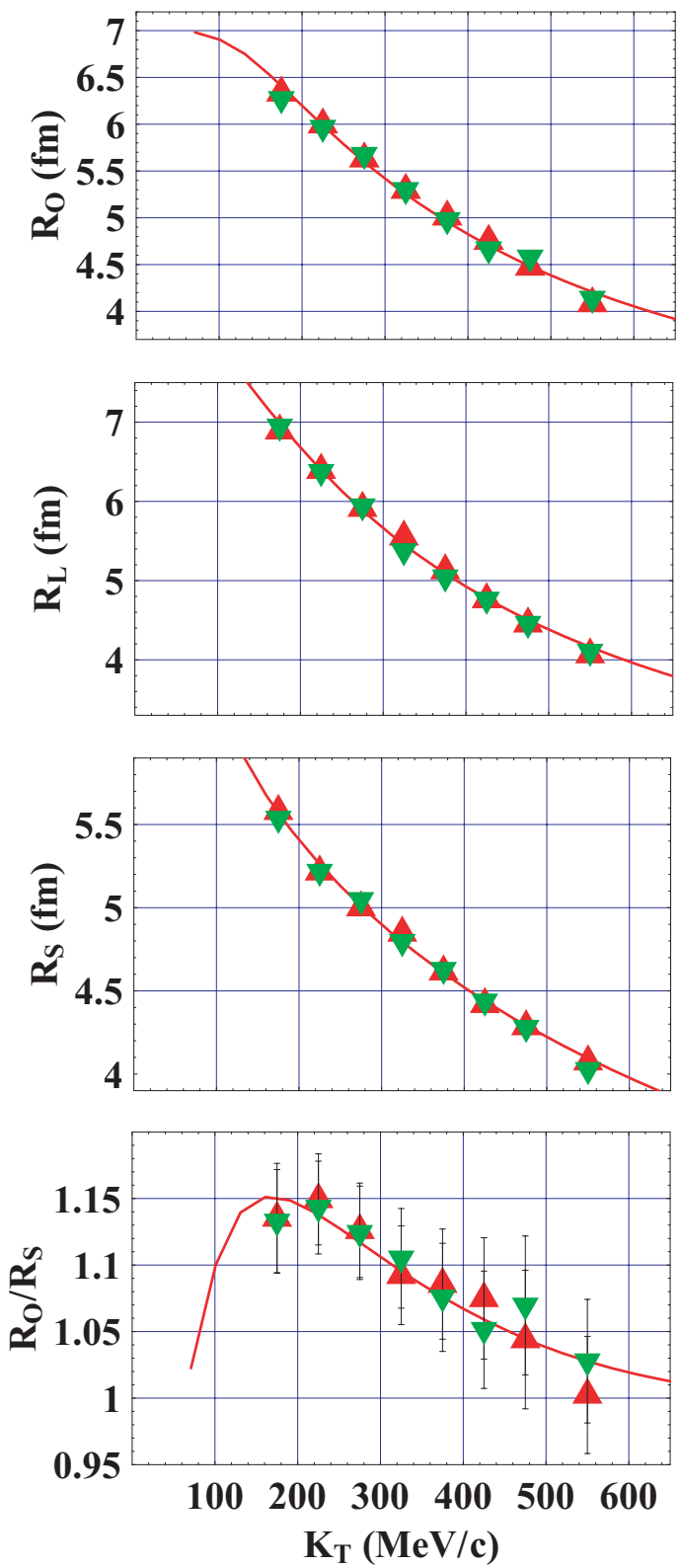

FIG. 2: (Color online) HBT Radii $R_{s}, R_{o}, R_{l}$ and the ratio $R_{o} / R_{S}$; Data [3]): $\nabla$ (green) $\Rightarrow \pi^{+} \pi^{+} ; \triangle$ (red) $\Rightarrow \pi^{-} \pi^{-}$. Curves: solid (red) full DWEF calculation.

$\tau_{0}$ to scale as the centrality-dependent number of participant particles to the one-third power: $N_{\text {part }}^{1 / 3}$. The values of $N_{\text {part }}$ are taken from Glauber-model calculations[5]. We assume that the optical potentials the same as that describing the medium environment in a central collision.

For the $\mathrm{Au}+\mathrm{Au}$ system, the value of $\Delta \tau$ is kept at the best fit value of Table I, because this is a dynamic quantity describing the proper-time duration during which pions are emitted in the collision.

For the $\mathrm{Cu}+\mathrm{Cu}$ system we also use participant scaling, but for this lighter system we have also assumed that the emission duration parameter $\Delta \tau$ scales as $A^{1 / 3}$, where $A$ is that atomic 


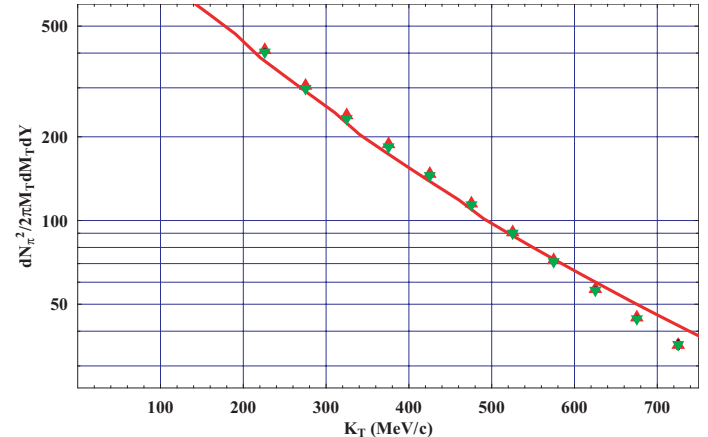

FIG. 3: (Color online) Pion momentum spectrum. Data [4]: $\nabla$ (green) $\Rightarrow \pi^{+} ; \triangle$ (red) $\Rightarrow \pi^{-}$. Solid (red) full DWEF calculation.

mass number of the colliding nuclei.

We see that for both systems, the scaled predictions of the DWEF model provide good qualitative predictions of the HBT radii for the four most central data sets, but show some problems, particularly in the prediction of $R_{S}$, for the two most peripheral collisions. This is to be expected, because it is not reasonable to expect that the same optical model parameters would describe the medium in central and the most peripheral collisions.

\section{CONCLUSION}

When we began this work, our expectation was that the imaginary part of the optical potential would be important for simulating pion absorption, while the real potential with its refractive effects was included mainly for formal reasons. To our surprise, when the fitting began the real potential grew deeper and deeper as the fit improved, until it was essentially as deep as the pion mass. This result suggested to us that the pion must be losing mass in the hot dense medium of the collision because chiral symmetry had been partially restored in the medium. Therefore, we gave the optical potential the momentum dependence that is consistent with chiral symmetry restoration. The result of this inclusion was impressive. Good fits to the STAR data, giving a chi-squared of about 4.7 per degree of freedom, were obtained. These fits are shown in Figs. 1 and 2 .

These results indicate that roughly half of the pions are absorbed while traversing the medium, while those surviving are emitted primarily from a localized "bright ring" near the outer surface of the medium. Moreover, the emerging pions must regain their mass by expending a sizable fraction of their kinetic energy in climbing out of the very deep well made by the real potential. When these effect are properly taken into account, the pion source size and emission duration are consistent with a QGP scenario. Further, in most lattice gauge studies of heated and compressed nuclear matter the chiral phase transition and the transition to a quark-gluon plasma occur under about the same conditions. Our inferred observation of a chiral phase transition at RHIC is therefore consistent with the presence of a quark-gluon plasma transition in RHIC
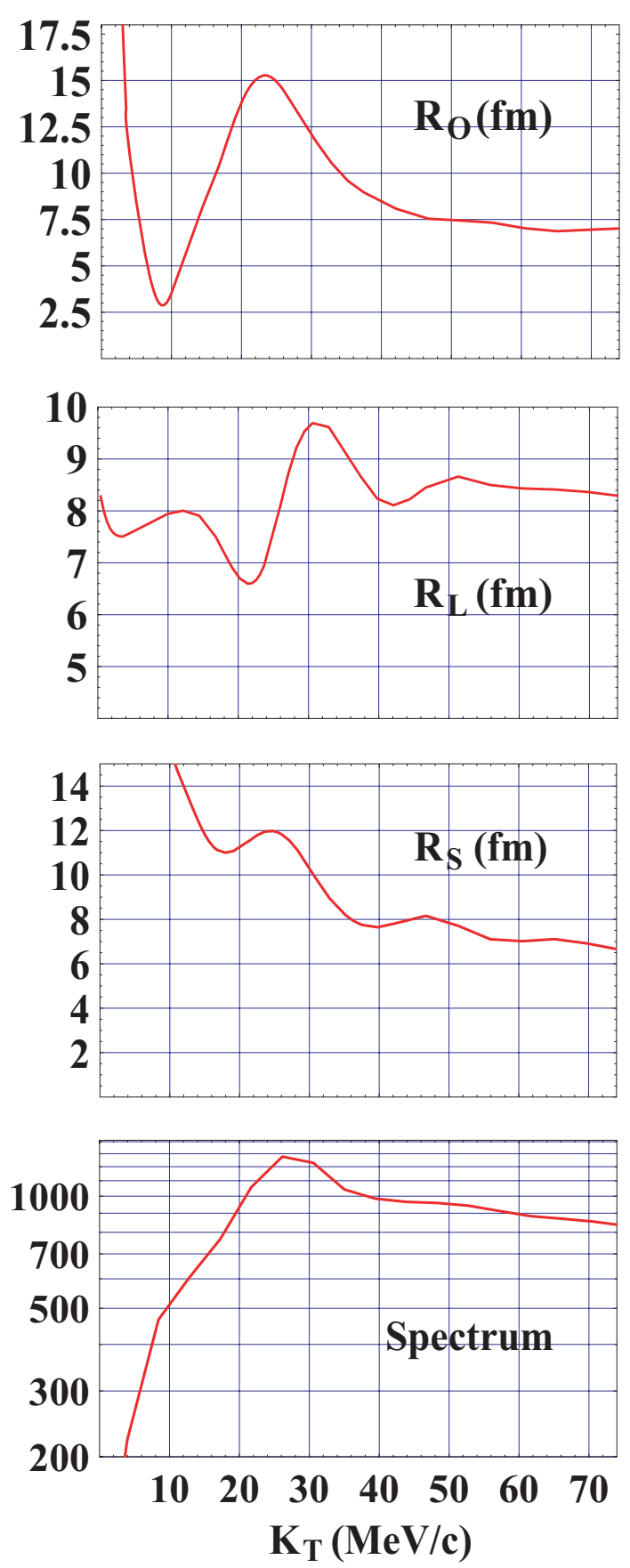

FIG. 4: (Color online) Low-momentum DWEF predictions of the fit listed in Table I.

collisions.

The question had been previously raised as to whether the DWEF model, despite its ability to accurately predict pion HBT radii and spectra including normalizations, is essentially a "surface model", which needs to describe the system only at the edge of the medium where the absorption ceases. We have investigated this question by setting the optical potential, the central ingredient of the DWEF description, to zero in the central radial region, and moving this "null zone" outward in 10 

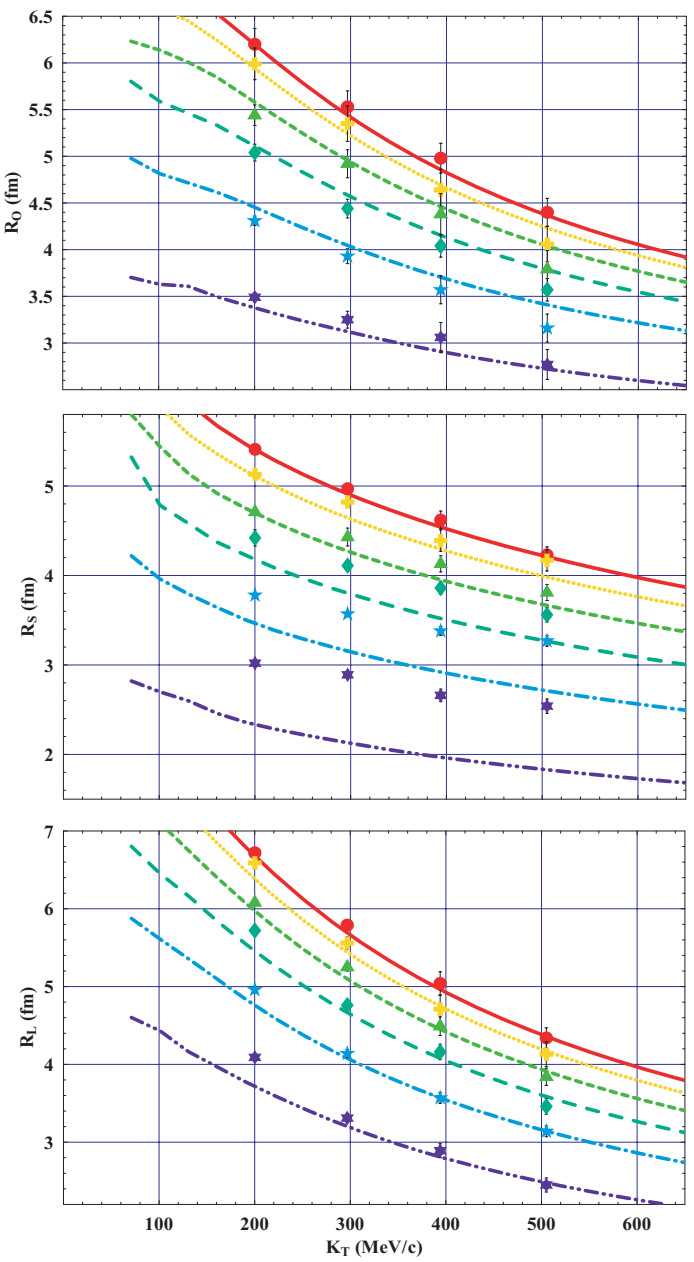

FIG. 5: (Color online) Centrality dependence of HBT radii in $\mathrm{Au}+\mathrm{Au}$ collisions. Curves are predictions from participant-scaled space-time parameters; data points are $\mathrm{Au}+\mathrm{Au}$ at $\sqrt{s_{N N}}=200 \mathrm{GeV}$ from [3] . Symbols and colors: $0-5 \%$ circle (red), $5-10 \%$ plus (yellow), $10-20 \%$ triangle (green), 20-30\% diamond (aqua), 30-50\% 5-star (blue), 50$80 \%$ 6-star (indigo).

concentric rings until the potential is zero everywhere. These results indicate that significant deviations from the baseline predictions of the data are observed after only one or two such steps away from the center of the collision, and that even setting the potential to zero only in the innermost ring zone produces significant depredation of the prediction of the $R_{O} / R_{S}$ ratio. We interpret this as indicating that the wavelike behavior of the pions probes the entire collision volume, not just the surface.

A question from the Workshop inquired whether it is appropriate for a dynamic system like a RHIC Au+Au collision to be modelled with a source that is instantaneous and an optical potential that is static. This appears to be a misunderstanding of the model The pion source described by the emission function emits pions over a modest time of about $6 \mathrm{fm} / \mathrm{c}$. The optical potential we use is intended to be the "true" time-varying optical potential averaged over this relatively short span of time. On other words, the optical potential is an average snap-
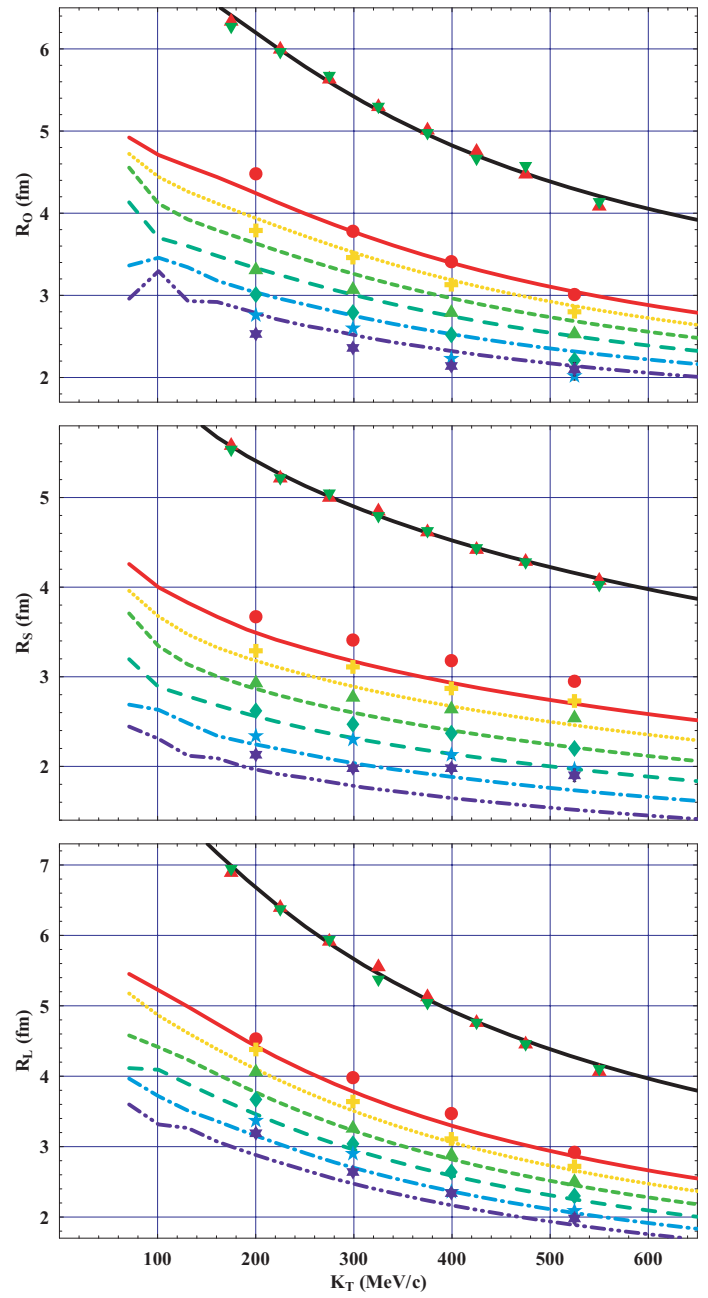

FIG. 6: (Color online) HBT Radii: Central $\mathrm{Cu}+\mathrm{Cu}(\sqrt{s}=200 \mathrm{GeV})$ predictions: $\mathrm{Au}+\mathrm{Au}$ - black solid; $\mathrm{Cu}+\mathrm{Cu}$ Centrality 0-10\% - red solid, $10-20 \%$ - yellow dot, $20-30 \%$ - green short-dash, 30-40\% aqua long-dash, $40-50 \%$ - blue dot-dash, $50-60 \%$ - violet dot-dotdash.

shot of interactions with the medium during the brief time that the pions are passing through the hot dense medium in which they are emitted.

Another Workshop question was whether the optical potential should have a direct connection to the emission function and should be computed from it rather than treated as an independent entity. The optical potential we use is not computed using the assumptions of thermodynamic equilibrium and detailed balance, which would give a definite relation between the source and the imaginary part of the optical potential. We have physical reasons for ignoring this implicit connection. In particular, we assume that the source of pions is some form of high density quark-gluon matter capable of emitting many pions. Suppose, for example, that a $q+g$ collision emits five pions. Our approach is to follow one or two of these pions. Then the $q+g \rightarrow 5 \pi$ term has some influence on the optical potential. E.g. the pion can collide with a quark and make 4 pions and a gluon. But the connection with the optical poten- 
tial is remote and indirect. In other words, the effects that are important in the source need not be important for the optical potential and the effects that are important for the optical potential need not be important for the source. This means that while there is a general underlying relation between the source $\mathrm{S}$ and the imaginary part of the optical potential $\mathrm{W}$, this relation may not be very useful in telling us what to use for either $\mathrm{S}$ or W. Therefore, we feel justified in treating the emission function and the optical potential as independent quantities requiring separate descriptions.

Another question from the Workshop is whether the behavior of this quasi-macroscopic system is stochastic, not coherent, so that interactions at different space-time regions of the medium during its evolution lead to random, not causally defined, phases for the quantum states. This would make it difficult to use the approximation of optical potential, at least, for fairly large particle momenta. Again, this appears to be a misunderstanding of the model We treat the sources as incoherent. However a pion emitted from such a source may undergo $0,1,2, \ldots$ rescatterings that lead to the same final state. Thus, the rescattering effects we treat are coherent. A formal derivation of this effect was presented by Gyulassy et al. in 1979[6] and is also in our recent paper[1]. Recently Kapusta[7] derived the same result.
In closing, we note that this work may represent the first direct observation of a chiral phase transition in a multiparticle system. Other experimental support of chiral symmetry restoration comes from the structure of highly excited states of the nucleon. Therefore, we have developed a new tool for relativistic heavy ion physics, which we plan to use for investigating the onset and properties of chiral symmetry restoration as a function of energy and centrality in relativistic heavy ion collisions, using data at the very wide range of energies and systems that has already been provided by experiments at the AGS, SPS, and RHIC.

\section{Acknowledgments}

We would like to express our gratitude the organizers of WPCF 2006. This work is partially supported by the USDOE grants Nos. DE-FG-02-97ER41014 and DE-FG-0297ER41020. GAM thanks LBL, TJNAF and BNL for their hospitality during the course of this work. We thank W. Busza, T. Csörgố, J. Draper, M. Lisa, M. Luzum, S. Pratt, J. Rafelski, S. Reddy, E. Shuryak and D. Son for useful discussions.
[1] Gerald A. Miller and John G. Cramer, J. Phys. G (Accepted for publication, 2007) [arXiv:nucl-th/0507004].

[2] John G. Cramer, Gerald A. Miller, Jackson M. S. Wu, and JinHee Yoon, Phys. Rev. Lett. 94, 102302 (2005).

[3] J. Adams, et al., Phys. Rev. Lett. 92, 112301 (2004).

[4] J. Adams, et al., Phys. Rev. C 71, 044906 (2005).
[5] M. Miller, "Measurement of Jets and Jet Quenching at RHIC", Yale University, Ph. D. Thesis (2001).

[6] M. Gyulassy, S. K. Kauffmann, and L. W. Wilson, Phys. Rev. C 20, 2267 (1979).

[7] J. I. Kapusta and Y. Li, arXiv:nucl-th/0503075. 